\title{
Extensão da Faixa Dinâmica de EDFAs: Análise da Influência da Alocação Aleatória de Canais
}

\author{
Adolfo F. Herbster, Júlio C. R. F. Oliveira, Juliano R. F. Oliveira e Aldário C. Bordonalli
}

\begin{abstract}
Resumo - Com base na estimação do ruído de emissão espontânea amplificada (ASE) dentro do ciclo de atuação do controle de ganho de amplificadores a fibra dopada com érbio (EDFA-CAG), torna-se possível a extensão da faixa dinâmica dos EDFAs. No entanto, esta estimação é baseada em um mapeamento prévio do acúmulo de ASE em função do ganho e da potência de entrada do EDFA, realizado para uma única alocação de canais. Como o EDFA possui um comportamento espectral extremamente dependente da alocação dos canais, neste trabalho será analisada, experimentalmente, a influência da alocação de canais no mapeamento da ASE.
\end{abstract}

Palavras-Chave - Amplificador à Fibra Dopada com Érbio (EDFA), Emissão Espontânea Amplificada (EEA), Correção de ASE.

\begin{abstract}
Based on estimation of noise from amplified spontaneous emission (ASE) within the cycle of action of automatic gain control of erbium doped amplifiers (EDFAAGC), its possible extend the dynamic range of EDFAs. However, this estimation is based on a survey of prior accumulation of ASE in terms of gain and power input of the EDFA, which is characterized for a single wavelength assignment. Since the EDFA has a very dependent spectral behavior with channels allocation, this paper experimentally analyzed the influence of the wavelength assignment in the mapping of the ASE.
\end{abstract}

Keywords-Erbium Doped Fiber Amplifier (EDFA), Amplifier Spontaneous Emission (ASE), ASE Correction.

\section{INTRODUÇÃO}

Sistemas de comunicações ópticos possuem alta capacidade de transmissão de informação por meio da multiplexação de vários canais de dados em uma única fibra.

Apesar de a fibra óptica apresentar baixa atenuação em torno de $1550 \mathrm{~nm}(\approx 0,2 \mathrm{~dB} / \mathrm{Km})$, para enlaces de médio/longo alcance, são necessários dispositivos que amplifiquem o sinal transmitido. Amplificadores ópticos representam uma tecnologia de alto desempenho devido à possibilidade de transmissão em longas distâncias sem conversão eletro-óptica. Os EDFAs e os amplificadores Raman representam as principais tecnologias de amplificação óptica, porém, devido a uma maior eficiência de conversão de bombeio, custo, versatilidade e praticidade os EDFAs representam a maioria absoluta (mais que $80 \%$ ) do mercado de amplificadores ópticos [2].

A. F. Herbster, J. C. R. F. de Oliveira e J. R. F. de Oliveira, (adolfo;julioc;jrfo)@cpqd.com.br, tel. +55-19-3705-6164, e A. C. Bordonalli, aldario@dmo.fee.unicamp.br, tel. +55-19-3521-3704.

Os autores agradecem ao apoio do CNPq, CAPES, FAPESP, FAEPEX/ UNICAMP e, em especial, do FUNTTEL e do CPqD, no âmbito do projeto de Pesquisa Aplicada - Rede Óptica Convergente (PA-ROC).
Os EDFAs possuem o mesmo princípio de amplificação dos lasers, baseando-se no processo de emissão estimulada capaz de produzir fótons de mesma fase e direção que o sinal óptico incidente. A amplificação ocorre quando está configurado o processo de inversão de população, em que o número de portadores do nível metaestável supera os portadores do nível fundamental. No entanto, além da amplificação do sinal de entrada via a emissão estimulada, também faz parte do processo de amplificação uma parcela de potência advinda da emissão espontânea amplificada (ASE), sendo esta oriunda do retorno espontâneo de portadores excitados ao nível fundamental. Assim, a saída de um EDFA é composta pelo sinal amplificado em conjunto com uma parcela de ruído (ASE). O nível de emissão de ASE é inversamente proporcional ao tempo de vida dos elétrons no nível metaestável [3].

Para o cálculo do ganho do EDFA, as técnicas de controle eletrônico se baseiam na monitoração das potências de entrada/saída obtidas dos fotodetectores, obtendo assim o ganho total. No entanto, a potência obtida não é composta apenas por sinal, e sim, principalmente na saída, pelo sinal e pela ASE gerada pelo EDFA devido ao processo de amplificação [4]. Desta forma, o controle de ganho realizado pela monitoração do ganho total estará tão impreciso quanto maior for a parcela de ASE, limitando, desta forma, a faixa dinâmica de controle dos EDFAs, não realizando o correto ajuste do ganho dos amplificadores que operam em redes ópticas reconfiguráveis.

Assim, torna-se necessário a utilização de técnicas para estimação da ASE em tempo real nos amplificadores, com o intuito de corrigir as medições de potência realizadas possibilitando o correto ajuste de ganho por canal. Um método de estimação em tempo real da ASE em EDFAs e sua correção por meio da atuação sob o bombeio foi proposto em [1]. Utilizando este método, é possível minimizar o erro entre o ganho real e o ganho alvo, tendo como consequiência a ampliação da faixa dinâmica dos EDFAs.

Entretanto, considerando que a composição da matriz hospedeira, a concentração de íons de $\mathrm{Er}^{3+}$ nas fibras dopadas e o número de canais do sinal óptico de entrada alteram o nível de emissão espontânea nestes amplificadores, torna-se necessário verificar o comportamento dos EDFAs dotados do método de correção da ASE.

Neste trabalho são apresentados os resultados obtidos para diversas formas de mapeamento de acordo com a alocação de canais do sinal óptico de entrada, com a intenção de encontrar uma forma adequada de realizar o mapeamento do erro em EDFAs sem degradar a nova faixa dinâmica obtida. A faixa dinâmica do amplificador corresponde à faixa de sinal óptico de entrada em que a variação do erro do ganho no canal seja 
menor que $1 \mathrm{~dB}$. Por mapeamento do erro entende-se a mensuração da parcela de ASE na saída do EDFA, esta dependente do carregamento de canais em sua entrada (espera-se) devido a não homogeneidade da fibra dopada com érbio.

A fundamentação teórica do problema é apresentada na seção II. O arranjo experimental é apresentado na seção III. O método de estimação e correção da ASE é apresentado na seção IV. Os resultados e conclusões dos dados obtidos são discutidos na seção V.

\section{PROCESSO DE AMPLIFICAÇÃO EM EDFA}

O ganho do sinal pode ser expresso em termos da inversão média dos íons de érbio ao longo da fibra. Assumindo uma perda nula, pode-se expressar a variação do sinal de entrada como [4]:

$$
\frac{d P_{s}(z)}{d z}=\left(N_{2}(z) \sigma_{s}^{e}-N_{1}(z) \sigma_{s}^{a}\right) P_{s}(z) \Gamma_{s}
$$

em que $\boldsymbol{P}_{\boldsymbol{s}}(\boldsymbol{z})$ representa o sinal incidente na posição $\boldsymbol{z}$ na fibra, $\boldsymbol{N}_{\mathbf{2}}(\mathbf{z})$ e $\boldsymbol{N}_{\mathbf{1}}(\mathbf{z})$ a densidade de população dos estados fundamentais e metaestável, $\boldsymbol{\sigma}_{\boldsymbol{s}}^{\boldsymbol{e}}$ e $\boldsymbol{\sigma}_{\boldsymbol{s}}^{\boldsymbol{a}}$ as seções de emissão e absorção, respectivamente, para uma fibra óptica dopada com érbio com comprimento $\boldsymbol{L}$ e com fator de sobreposição $\boldsymbol{\Gamma}_{\boldsymbol{s}}$. O ganho do sinal é obtido integrando-se a equação (1):

$$
G=\int_{0}^{L}\left(N_{2}(z) \sigma_{s}^{e}-N_{1}(z) \sigma_{s}^{a}\right) \Gamma_{s} d z
$$

A equação (2) sugere que o ganho seja uma função complexa do formato de inversão ao longo do comprimento da fibra. Na verdade, o ganho pode ser obtido pela inversão média, considerando a inexistência de variações bruscas de população dentro da fibra. Desta forma, definindo-se:

$$
\begin{aligned}
& \bar{N}_{2}=\frac{1}{L} \int_{0}^{L} N_{2}(z) d z \\
& \bar{N}_{1}=\frac{1}{L} \int_{0}^{L} N_{1}(z) d z
\end{aligned}
$$

A equação pode ser simplificada como:

$$
G=\exp \left[\left(\bar{N}_{2} \sigma_{s}^{e}-\bar{N}_{1} \sigma_{s}^{a}\right) \Gamma_{s} L\right]
$$

Para uma freqüência $\boldsymbol{v}$ em particular:

$$
G(v)=\exp \left[\left(\bar{N}_{2} \sigma_{s}^{e}(v)-\bar{N}_{1} \sigma_{s}^{a}(v)\right) \Gamma_{s} L\right]
$$

$\boldsymbol{G}(\boldsymbol{v})$ na equação (6) representa o ganho da fibra e significa o ganho óptico derivado da fibra dopada com érbio. O ganho efetivo para o amplificador pode ser descrito por:

$$
G_{e f}=\frac{G(v)}{\Gamma_{1} \Gamma_{2}}
$$

em que $\boldsymbol{\Gamma}_{\mathbf{1}}$ e $\boldsymbol{\Gamma}_{\mathbf{2}}$ representam as perdas de conexão de entrada (antes da fibra dopada com érbio (FDE)) e de saída (após a FDE) respectivamente.

A potência de ASE na saída do EDFA é função da frequiência óptica $\boldsymbol{v}$ (com faixa de medida de $\boldsymbol{\Delta} \boldsymbol{v}$ ), com ganho $\boldsymbol{G}$

$$
P_{A S E}(v)=2 \eta_{s p}(G-1) h v \Delta v
$$

na qual $\boldsymbol{\eta}_{\boldsymbol{s p}}$ é o fator de emissão espontâneo definido por [5].

$$
\eta_{s p}=\frac{\sigma_{e} N_{2}}{\sigma_{e} N_{2}-\sigma_{a} N_{1}}
$$

$\mathrm{Na}$ prática, os EDFA estabelecem ganhos elevados para amplificar sinais baixos. Considera-se $\boldsymbol{G}(\boldsymbol{v}) \gg \mathbf{1}$ e $\boldsymbol{\eta}_{\boldsymbol{s p}} \approx \mathbf{1}$. Nestas condições, a equação (8) pode ser expressa por:

$$
P_{A S E}(v) \approx G(v) h v \Delta v
$$

Verifica-se em condições de saturação, por exemplo, quando a potência óptica de entrada é elevada e o ganho é baixo $\left(\boldsymbol{\eta}_{\boldsymbol{s} \boldsymbol{p}}<1\right)$, que em tais condições a contribuição da ASE na potência total de saída é pequena e pode ser desconsiderada [6].

A potência de ASE, considerando as perdas ao longo da cadeia de componentes do amplificador, é expressa por:

$$
P_{A S E}(v)=\frac{2 G(v) h v \Delta v}{\Gamma_{2}}
$$

A potência total do ASE $\left(\boldsymbol{P}_{\boldsymbol{A} \boldsymbol{S} E}^{T}\right)$ é obtida realizando a integração da equação (11) ao longo da faixa de emissão:

$$
P_{A S E}^{T}=\frac{2}{\Gamma_{2}} \int_{v_{1}}^{v_{2}} G(v) h v d v
$$

sendo $\boldsymbol{v}_{\mathbf{1}}$ e $\boldsymbol{v}_{\mathbf{2}}$ os limites de operação do amplificador em Hz. Observa-se a relação decrescente entre a figura de ruído e a perda $\boldsymbol{\Gamma}_{\mathbf{2}}$, sendo o ganho do estágio elevado.

A equação (12) pode ser simplificada substituindo o valor de $\boldsymbol{G}(\boldsymbol{v})$ por sua média $\overline{\boldsymbol{G}}$ ao longo da faixa de operação do amplificador. Esta aproximação é válida para amplificadores que contenham um filtro óptico (Gain-Flattening Filter GFF) após a FDE. O erro introduzido por esta simplificação é corrigido pelo parâmetro $\boldsymbol{\alpha}$, dessa forma a equação (12) é escrita na forma:

$$
P_{A S E}^{T}=\frac{2 \alpha \bar{G}}{\Gamma_{2}} \int_{v_{1}}^{v_{2}} h v d v
$$

sendo esta equação fundamental utilizada para correção da ASE. De acordo com a equação (13), a potência total de ASE é dependente apenas do ganho e da faixa do sinal óptico incidente.

Relatos experimentais [7] mostram que o Spectral Hole Burning (SHB) reduz a potência de ASE na freqüência do 
sinal óptico incidente. Entretanto, a diferença entre os dois níveis de ASE ocorre principalmente em comprimentos de onda que estão relativamente distantes do comprimento de onda do sinal óptico de entrada [6], com potência de ASE total crescente com o aumento da potência do sinal óptico incidente, como ilustrado na Figura 1.

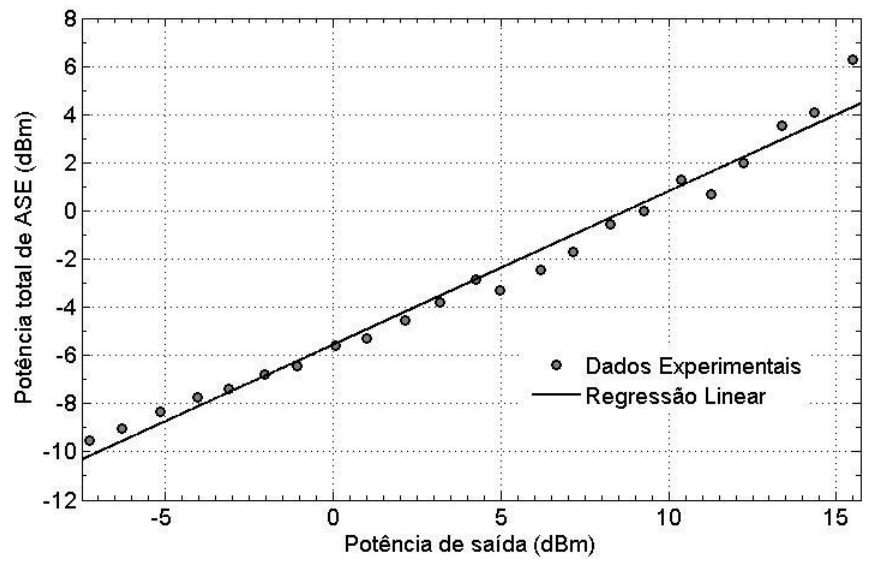

Figura 1: Potência de ASE medida versus potência de saída óptica.

Considerando a dependência linear da potência total da ASE com a potência de saída é adicionado o fator $\boldsymbol{k}_{\mathbf{1}} \boldsymbol{P}_{\text {out }}$ de correção [5], sendo $\boldsymbol{k}_{\mathbf{1}}$ uma constante de proporção e $\boldsymbol{P}_{\text {out }}$ a potência de saída do amplificador. A equação (13) é modificada para a forma:

$$
P_{A S E}^{T}=\frac{2 \alpha \bar{G}}{\Gamma_{2}} \int_{v_{1}}^{v_{2}} h v d v+k_{1} P_{o u t}
$$

A potência da ASE total é expressa por:

$$
P_{A S E}^{T}=k_{2} \bar{G}+k_{1} P_{\text {out }}
$$

em que,

$$
k_{2}=\frac{2 \alpha}{\Gamma_{2}} \int_{v_{1}}^{v_{2}} h v d v
$$

A equação (15) quantifica o comportamento da ASE ao longo do processo de amplificação no EDFA. A proporção mantida com o ganho e a potência de saída é originada do bombeio necessário para obter o ganho especificado, que com uma mesma potência óptica incidente, resulta em uma potência óptica de saída.

Fixando-se o nível de bombeio e elevando-se o nível de sinal incidente, o valor da ASE total diminui, devido à saturação do amplificador pelo sinal incidente, como ilustra a Figura 2.

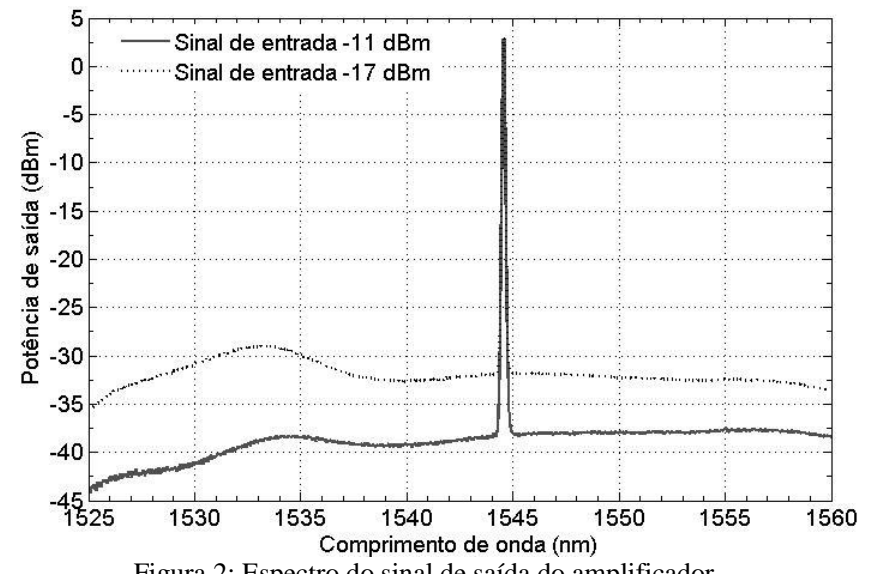

Figura 2: Espectro do sinal de saída do amplificador.

Devido ao espalhamento não-homogêneo e interações entre íons devido a formações de aglomerados de $\mathrm{Er}^{3+}$ na matriz hospedeira [8], o número de canais influencia o nível de potência de ASE no espectro, como pode ser ilustrado na Figura 3. Com o aumento do número de canais ao longo do espectro óptico há uma diminuição da emissão espontânea de fótons, nos comprimentos de ondas ocupados, devido ao aumento da emissão estimulada gerada pelos canais ao longo da banda (Figura 3).

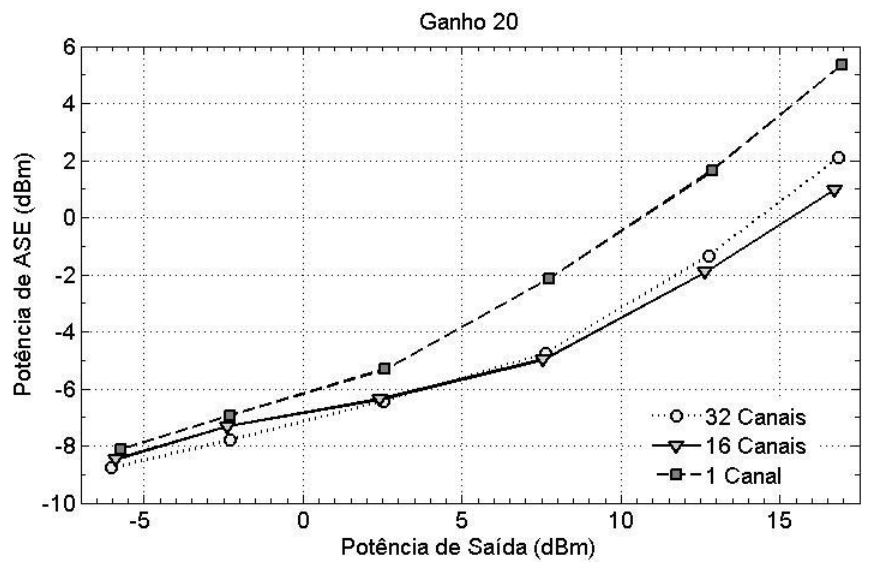

Figura 3: Nível de potência de ASE de acordo com o número de canais contidos no sinal óptico de entrada.

\section{CONFIGURAÇÃO EXPERIMENTAL}

\section{A - Configuração do EDFA utilizado}

A Figura 4 ilustra o EDFA-CAG utilizado no arranjo experimental. Este amplificador é composto por elementos básicos de um sistema de amplificação óptica, são eles: a fibra dopada com érbio (FDE), acopladores de sinal de entrada/saída, isoladores e acopladores WDM.

A fim de realizar a leitura do sinal óptico incidente, uma parcela do sinal incidente é retirada pelo acoplador de entrada (95-5\%) e convertida em corrente elétrica no fotodetector de entrada (PD1). O sinal produzido pelo fotodetector é adquirido pelo conversor analógico/digital e enviado para o controlador digital de sinais (Digital Signal Controller DSC). Este DSC realiza o controle de ganho com supressão de transiente utilizando aritmética de ponto - fixo. Neste tipo 
de aritmética, evita-se utilizar expressões numéricas com funções transcendentais, impactando no modo comum de compensação de ASE. Buscou-se a estimação do erro no controle automático de ganho, por realizar apenas a operação de subtração na variável de ganho.

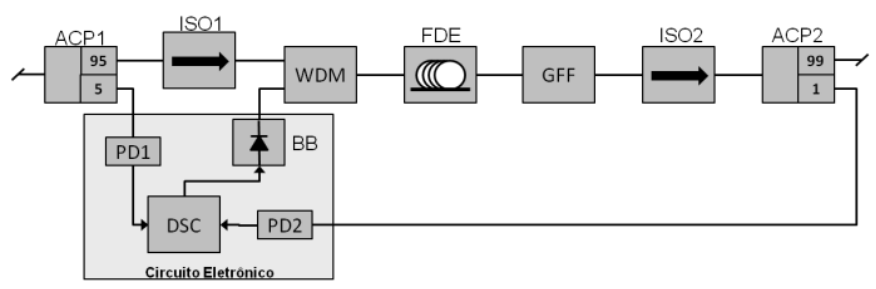

Figura 4:Controle de ganho eletrônico realimentado aplicado a um EDFA.

Similarmente ao sinal incidente, uma parcela do sinal de saída é retirada pelo acoplador de saída (ACP2), convertida em corrente elétrica no fotodetector de saída (PD2) e enviada ao DSC. Com as informações de potência de entrada e saída, o ganho é calculado no DSC, ajustando-se a potência de bombeio por meio do controlador digital, garantindo a manutenção do ganho desejado. A potência de saída máxima é limitada pelo comprimento da FDE e pelo laser de bombeio (BB). O EDFA utilizado, onde é aplicada a tabela de mapeamento, possui uma potência de saída máxima de 16 dBm e mínima de $-10 \mathrm{dBm}$, com faixa dinâmica ideal de 26 $\mathrm{dB}$.

Como citado anteriormente, os fotodetectores não são capazes de determinar qual a parcela do sinal detectado é composta por ASE, e qual é composta por sinal. Desta forma, o ganho calculado (ganho real) não representa o ganho alvo, realizando uma amplificação do sinal de forma imprecisa, aumentando esta imprecisão com a redução da potência de entrada.

\section{$B$ - Arranjo Experimental}

A fim de analisar a influência da ASE na potência de saída e realizar a correção da potência de ASE do sinal óptico amplificado, utiliza-se o arranjo experimental ilustrado na Figura 5. O arranjo consiste em controlar a inserção e remoção de canais (entre 1 e 32 canais), utilizando uma chave óptica (CO). Foram utilizados canais com comprimentos de onda localizados na banda $\mathrm{C}$ de acordo com a grade ITU. 32 canais correspondem aos comprimentos de onda entre $\mathrm{C} 23 \mathrm{e}$ C54, 16 canais entre os canais C23 e C53 com apenas numeração ímpar. Um laser sintonizável (LS) é utilizado como o canal óptico sobrevivente, compondo o sinal óptico de entrada antes e após a remoção dos canais da bancada de lasers (BL), realizada pela chave óptica. O sinal resultante, composto pelo canal sobrevivente e pelos canais da bancada de lasers, é obtido por meio do acoplador ACP1. Em seguida o sinal resultante passa por um atenuador óptico variável (ATT) de bancada, ajustando a potência incidente no EDFA. Para realizar a medida de potência de forma automática, utilizou-se um medidor de potência óptica (MP), obtendo-se uma amostra do sinal óptico por meio do acoplador ACP2. Após passar pelo EDFA, o sinal óptico amplificado é observado em um analisador de espectro óptico (ASO), e em um osciloscópio (OSC), após a conversão por meio de um fotodetector (FD) do sinal óptico em sinal elétrico.

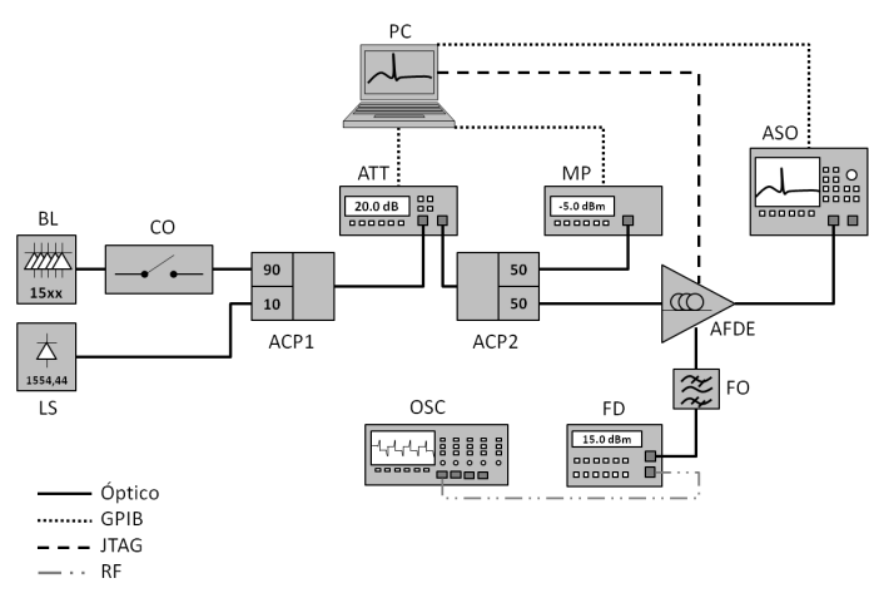

Figura 5: Arranjo experimental utilizado para obtenção dos resultados.

A caracterização é realizada uma única vez, para todas as condições de sinal. No final deste processo, uma tabela de coeficientes é gerada e adicionada ao algoritmo de controle de ganho do EDFA.

\section{C-Medição do Erro no Controle Automático de Ganho}

A medição do ganho em amplificadores utilizando um analisador de espectro óptico é realizada comparando-se os níveis do sinal óptico de entrada e saída, enquanto a medição da figura de ruído necessita da determinação do nível de ASE gerada pelo EDFA, refletido na razão entre a relação sinalruído (SNR) de saída e entrada [4,9]. O valor de SNR expressa a qualidade do sinal e é determinado em termos dos níveis de sinal e ruído na corrente gerada no fotodetector disposto no percurso do sinal $[3,4]$.

Entretanto, no ASO, o sinal óptico amplificado se sobrepõe ao valor da ASE, impossibilitando a medição em separado dos níveis de sinal e de ASE. Há diversos métodos que possibilitam a medição do nível de ASE, dentre os quais [9]:

- Subtração de fonte;

- Extinção no domínio do tempo;

- Extinção por polarização;

- Fonte reduzida;

- Substituição do sinal;

- ASE contra-propagante [10];

Neste trabalho, o erro no controle devido à parcela de ASE foi avaliado por meio do cálculo do erro do ganho, obtido por meio da diferença entre o ganho referência e o ganho real. Este erro foi obtido calculando a diferença entre o nível do canal incidente (conhecido previamente) adicionado do ganho alvo, e o nível do canal na saída obtido por meio do ASO. Conhecida esta diferença, para cada ganho e potência de entrada a qual se deseja operar com o EDFA, o cálculo do ganho passa a ser realizado compensando este valor por meio do acréscimo ou uma redução na potência de bombeio. 


\section{RESULTADOS E ANÁLISES}

Os resultados foram obtidos por meio do arranjo experimental apresentado na Figura 5. A metodologia adotada consiste em acoplar à entrada do EDFA um canal no comprimento de onda de $1544,54 \mathrm{~nm}$, com potência ajustada pelo atenuador óptico variável, verificando em seguida, por meio do ASO, o espectro de saída do EDFA, obtendo assim o ganho real do canal. São consideradas diversas formas de preenchimento do espectro óptico (um canal, 16 canais e 32 canais espaçados igualmente de forma a ocupar toda a banda C).

O experimento consiste em uma etapa de verificação da melhor forma de mapear o erro devido à parcela de ASE contida no sinal de saída ( 1,16 ou 32 canais), e em uma etapa subsequiente que consiste em verificar a extensão da faixa dinâmica do amplificador com os resultados dos mapeamentos realizados.

Por meio de um sistema de caracterização completamente automatizado, o ganho alvo foi variado entre 5 e $25 \mathrm{~dB}$ com 1 $\mathrm{dB}$ de espaçamento, sendo, para cada ganho, variada a potência de entrada entre $-26 \mathrm{dBm}$ e $-3 \mathrm{dBm}$. O erro associado à parcela de ASE foi mapeado para cada ganho e cada potência de entrada, coletando-se o espectro de saída em cada uma destas condições e aplicando a técnica de medição de ASE descrita na seção anterior.

\section{A-Mapeamento do erro}

O espectro de emissão de ASE é caracterizado por fatores como composição da matriz hospedeira, temperatura, inversão de população, alocação e potências dos canais ópticos ao longo do espectro. Na análise em questão, os fatores estáticos, ou seja, que condizem ao processo de fabricação da fibra e ao ambiente são desconsiderados, considerando-se os fatores relacionados aos canais ópticos incidentes.

É verificado como a composição de canais do sinal óptico incidente pode afetar o mapeamento do erro do ganho ocasionado pelo acúmulo de ASE na saída do EDFA. Com o ganho do amplificador (EDFA - CAG) ajustado para $20 \mathrm{~dB}$ foi realizado o mapeamento do erro com apenas um canal $(1554,54 \mathrm{~nm})$ no sinal óptico incidente. Após, foi adicionada a tabela gerada pelo mapeamento do erro com 1 canal no algoritmo de controle de ganho do EDFA, verificando-se o resultado obtido para uma entrada composta por 1 canal, 16 canais e 32 canais no sinal óptico incidente. Os resultados estão ilustrados na Figura 6 (b).

De forma análoga ao procedimento anterior, o mapeamento foi realizado para um sinal óptico incidente contendo 16 e 32 canais. Os resultados são apresentados na Figura 6(c), para 16 canais, e na Figura 6(d) para 32 canais.

Considerando os resultados apresentados, verifica-se que a extensão da faixa dinâmica do amplificador (Figura 6) independe do número de canais utilizados para gerar a tabela de erro. A princípio, conforme discutido anteriormente (Figura 3), carregamentos distintos induzem a comportamentos espectrais distintos, já que influenciam o nível de ASE.
Porém, verificamos que devido à utilização de GFF no projeto óptico (aplainando o ganho do EDFA), e às perdas geradas pelo processo de mapeamento e controle, o mapeamento torna-se independente do número de canais contidos no sinal óptico incidente. Verifica-se que diferença entre a faixa dinâmica das formas de mapeamento não é maior que 3,1 dB (Tabela 1 - Carregamento de 32 canais).

TABELA 1: ANÁLISE DA FAIXA DinÂMICA

\begin{tabular}{ccccc}
\hline $\begin{array}{c}\text { Número } \\
\text { de Canais }\end{array}$ & $\begin{array}{l}\text { Sem } \\
\text { Correção }\end{array}$ & $\begin{array}{c}\text { Carregamento } \\
\text { de } 1 \text { canal }\end{array}$ & $\begin{array}{l}\text { Carregamento } \\
\text { de 16 canais }\end{array}$ & $\begin{array}{l}\text { Carregamento } \\
\text { de 32 canais }\end{array}$ \\
\hline 1 & 7 & 19,2 & 20 & 18,9 \\
\hline 16 & 12 & 20,1 & 19,2 & 20,2 \\
\hline 32 & 14,5 & 20,8 & 23 & 22 \\
\hline
\end{tabular}
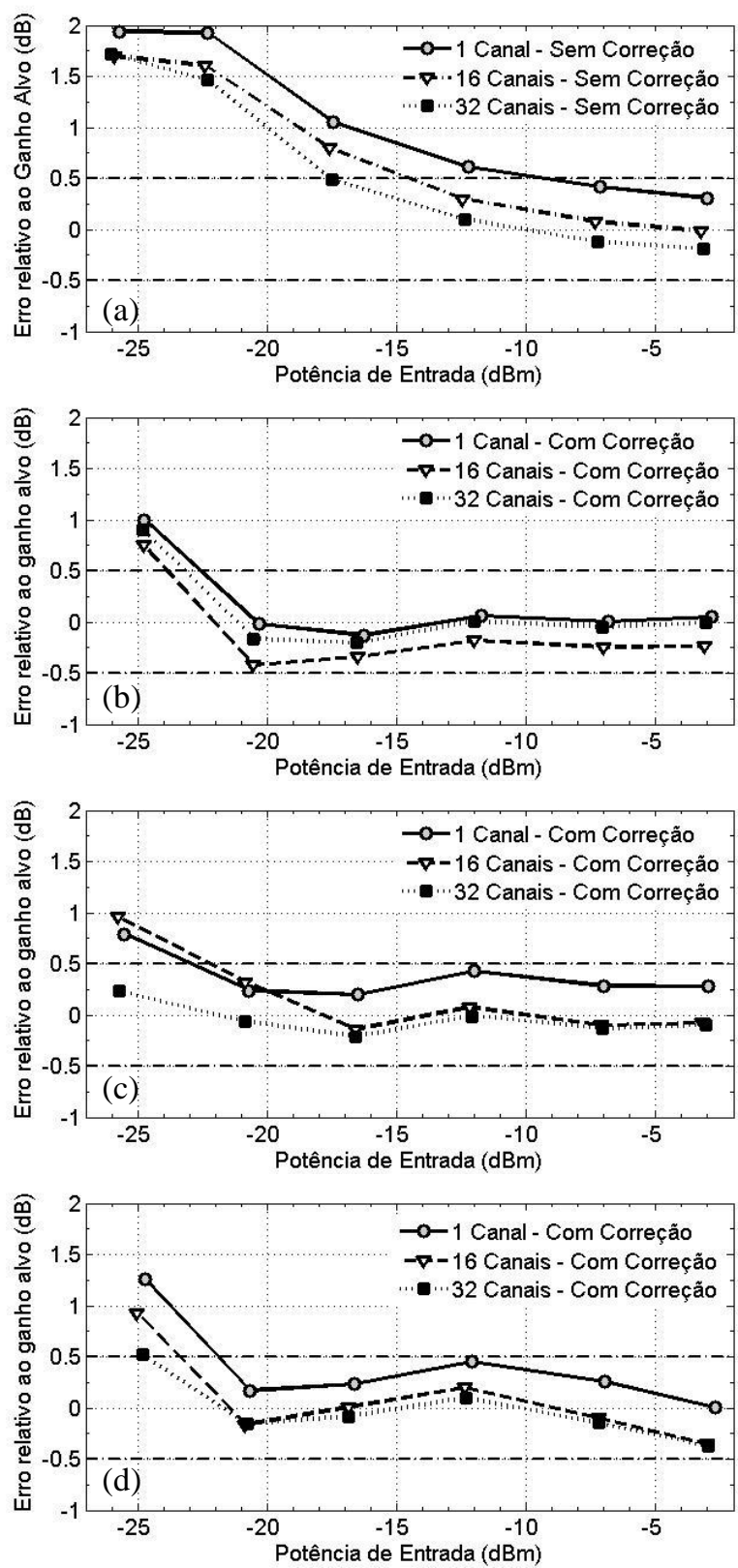

Figura 6: Erro relativo ao ganho de $20 \mathrm{~dB}$ para diferentes carregamentos de canais: (a) Sem correção de ASE; (b) Com tabela de correção para carregamento de 1 canal; (c) Com tabela de correção para carregamento de 16 canais; (d) Com tabela de correção para carregamento de 32 canais. 
Com base nos resultados obtidos, o mapeamento do erro com 1 canal pode ser utilizado como padrão para levantamento do erro do ganho induzido pela ASE, tornando o experimento mais simples e com um resultado confiável independente do carregamento do EDFA.

\section{B - Extensão da Faixa Dinâmica}

Com a tabela de mapeamento obtida na seção anterior utilizando apenas um canal, o efeito da correção é realizado para toda a faixa do amplificador com $20 \mathrm{~dB}$ de ganho. A Figura 7 ilustra os resultados. Observa-se que, para um ganho de $20 \mathrm{~dB}$, a faixa dinâmica ideal é $26 \mathrm{~dB}$, ou seja, o canal não varia mais que $1 \mathrm{~dB}$ do ganho de referência ao longo de 26 dB.

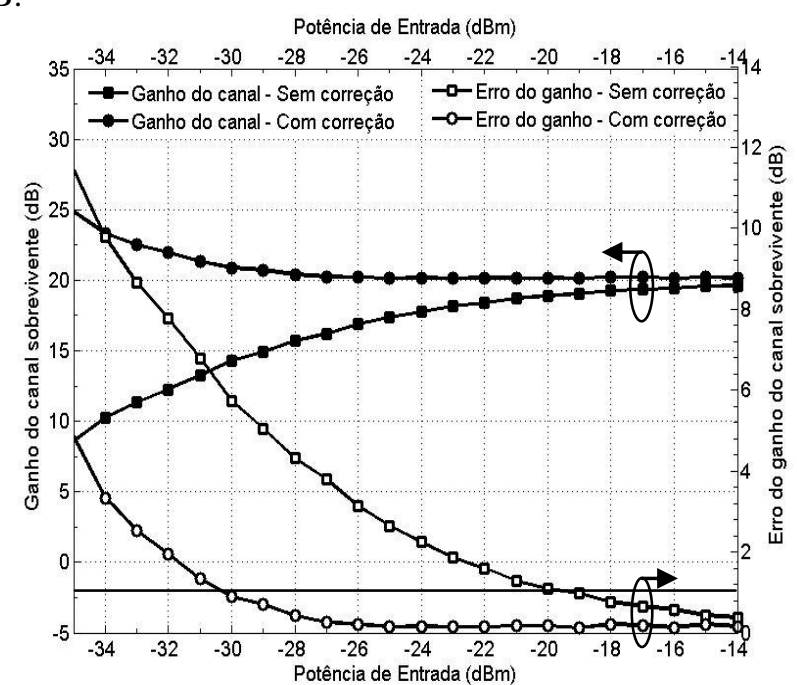

Figura 7: Resultado da análise de operação do EDFA com ganho de $20 \mathrm{~dB}$. (Para sinais de potência entre $-14 \mathrm{dBm}$ e $-4 \mathrm{dBm}$ de entrada, o erro do ganho é menor que $0,5 \mathrm{~dB}$ ).

Analisando a Figura 7, verifica-se que sem a correção de ASE a faixa dinâmica do EDFA-CAG é limitada a $15 \mathrm{~dB}$. No entanto, aplicando-se o método de correção proposto, a faixa dinâmica obtida é de $26 \mathrm{~dB}$, se igualando à faixa dinâmica ideal, e, conseqüentemente, eliminando o efeito da parcela de ASE na saída do EDFA.

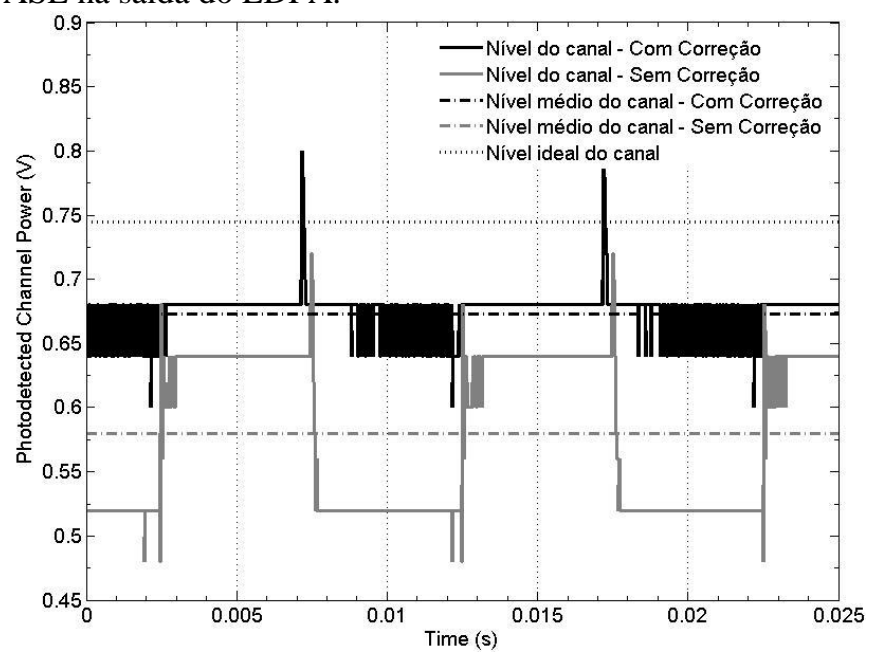

Figura 8: Resultado da análise temporal do EDFA com ganho fixo de $20 \mathrm{~dB}$ frente a uma variação de sinal de $9 \mathrm{~dB}$.
Por fim, a Figura 8 ilustra a análise temporal do canal sobrevivente. Para obtenção dos resultados foram inseridos e retirados sete canais, promovendo uma variação de $9 \mathrm{~dB}$ na potência de entrada do EDFA. Verifica-se (Figura 8) que o nível médio do canal fica a 1,09 dB abaixo do nível ideal do canal, sem utilização da correção de ASE. Por outro lado, ao utilizar o método de correção de ASE o nível do canal médio se torna maior, ficando $0,44 \mathrm{~dB}$ abaixo do nível ideal do canal, demonstrando assim o efeito da correção da ASE.

\section{CONCLUSÃO}

Foi constituída e demonstrada, experimentalmente, uma técnica de controle eletrônica de ganho com correção de ASE aplicada a EDFAs. Por meio de análises experimentais foi confirmada, para diversos valores de ganho fixos, a ampliação da faixa dinâmica proporcionada pela correção de ASE, comparando, no mesmo EDFA, o controle de ganho com e sem a correção aplicada. A dependência do mapeamento de ASE com o carregamento do EDFA foi pela primeira vez analisada. Concluímos que esta independe do carregamento de canais, sendo então, por razões práticas recomendado o mapeamento com 1 canal.

Por meio da ampliação da faixa dinâmica, obtida via a inserção do método de correção de ASE no controle eletrônico de ganho, a aplicação dos EDFA que fazem uso deste controle em redes ópticas reconfiguráveis é generalizada para operação na banda $\mathrm{C}$, suportando uma variação de sinal de entrada maior que $20 \mathrm{~dB}$.

\section{REFERÊNCIAS}

[1] A. F. Herbster, J. C. F. de Oliveira e A. Bordonalli, "Extensão da Faixa Dinâmica por meio da Correção da ASE aplicada a EDFA's em Redes Ópticas WDM," Momag, Setembro de 2008.

[2] P. C. Becker, N. A. Olsson e J. R. Simpson, Erbium-Doped Fiber Amplifiers: Fundamentals and Technology, Academic Press, 1999.

[3] D. Emmanuel, Erbium-doped fiber amplifiers : principles and applications, New York : Wiley Interscience, 1994.

[4] A. Srivastava, What is Next for Optical Amplifiers?, Oneterabit presentation, 2006.

[5] L. Qiao e P. J. Vella, "ASE Analysis and Correction for EDFA Automatic Control," Journal of Lightwave Technology, v. 3, pp. 771-778, Março de 2007.

[6] D. M. Baney, P. Gallion e R. S. Tucker, "Theory and Measurement Techniques for the Noise Figure of Optical Amplifiers," Optical Fiber Technology, v. 6, pp. 122-154, Abril de 2000.

[7] Y. Sato, Y. Yamabayashi e Z. Aida, "Noise Figure Monitoring of Optical Amplifiers via Backward Amplified Spontaneous Emission," IEEE Photonics Technology Letters, v. 6, Fevereiro de 1994.

[8] G. P. Agrawal, Telecomunication Systems. New Jersey : John Wiley \& Sons, 2005.

[9] E. Desurvire, J. L. Zyskind e J. R. Simpson, "Spectral gain hole-burning at 1.53 um in erbium-doped fiber amplifiers," IEEE Photon. Technology Letters, v. 2, pp. 246-248, Abril de 1990.

[10] J. C. Wright, "Up-conversion and excited state energy tranfeer in rareearth doped materials," Radiationless Processes in Molecules and Condensed Phases, v.15 of Topics in Applied Physics, pp. 239-295, 1976. 